\title{
Image processing for resonance frequency mapping in atomic force modulation microscopy
}

\author{
R. Arinéro, ${ }^{a)}$ G. Lévêque, P. Girard, and J. Y. Ferrandis \\ Laboratoire d'Analyse des Interfaces et de Nanophysique (LAIN), UMR CNRS 5011, \\ Université Montpellier II, CC 082, Place E. Bataillon, 34095 Montpellier Cedex, France
}

(Received 26 June 2006; accepted 18 December 2006; published online 8 February 2007)

\begin{abstract}
It has been demonstrated that the resonance frequency of the cantilever in atomic force modulation microscopy can be used to study local mechanical properties. We developed a numerical method to achieve mapping of the resonance frequency without significant modification of the device. By making the assumption that the resonance spectrum can be approximated by a Lorentzian curve, we established analytical expressions of the resonance frequency and the width of the curve (damping) depending on the real and imaginary parts of the vibration at a single frequency. Then, resonance frequency and damping images were produced from the recording of both the real and imaginary part images of the complex amplitude. The results on a standard high-impact polystyrene sample are shown. (C) 2007 American Institute of Physics. [DOI: 10.1063/1.2432264]
\end{abstract}

\section{INTRODUCTION}

The production of (visco)elastic images at the nanometer scale has become a fairly routine operation: either in atomic force modulation microscopy ${ }^{1}$ by recording amplitude (or phase) variations or in the tapping ${ }^{\circledR}$ mode $^{2}$ by observing a phase contrast, which is still poorly understood. Currently, the challenge is to quantify these images in terms of viscoelastic moduli.

A method was commercialized a few years ago, under the name of "force volume imaging," 3 that can be used to record the force-distance curves $F(d)$ on the entire investigated zone. The elastic penetration of the tip into the sample can be deduced thanks to a simple comparison with an $F(d)$ curve acquired on an infinitely stiff sample with respect to the tip. ${ }^{4}$ It is then possible, using an appropriate contact mechanics theory, to quantify the produced images. ${ }^{5}$ Because the data on cantilever stiffness and tip shape are not reliable enough, this last method applies only to some particular cases within suitable approximations. These cases are those for which the tip penetrates strongly into the sample (as for a nanoindentation experiment), so that the apex contribution to the elastic deformations is neglected in comparison with the rest of the tip, whose shape is more easily estimated.

Another method combines nanoindentation and force modulation microscopy: ${ }^{6}$ a diamond tip with a curvature radius of $250-600 \mathrm{~nm}$ is simultaneously submitted to a constant force of about $2 \mu \mathrm{N}$ and a sinusoidal force of about $1 \mu \mathrm{N}$. The excitation frequency is very low $(10-250 \mathrm{~Hz})$. The amplitude of displacement of the tip is small (linear regime) and, depending on the sample stiffness, it varies from 0.5 to $2 \mathrm{~nm}$. Both amplitude and phase measurements at the modulation frequency $f_{\text {mod }}$ are easily related to the complex writing of the contact stiffness. Taking into account

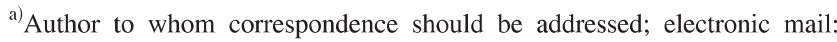
arinero@1ain.univ-montp2.fr
}

the magnitude of the applied force in comparison with the adhesion force, the Hertz contact model can be used to produce images of the so-called storage modulus $E^{\prime}\left(f_{\text {mod }}\right)$ and loss modulus $E^{\prime \prime}\left(f_{\text {mod }}\right)$. This method has recently been commercialized under the name of nanoDMA ${ }^{\mathrm{TM}}$ (nano dynamic mechanical analysis ${ }^{7}$ ).

Another interesting method was proposed by Anczykowski et al. ${ }^{8}$ This approach consists of analyzing the energy flow in the atomic force microscopy (AFM) system in different dynamic modes, particularly in the tapping ${ }^{\circledR}$ mode. Based on the hypothesis of a harmonic displacement of the tip, it provides an easy-to-use result. Indeed, the energy dissipated by tip-sample interactions can be directly obtained by measuring quantities such as oscillation amplitude, frequency, phase shift, etc. Furthermore, the authors proposed a setup to map these local variations of dissipation while scanning a sample surface. Applied to a polymer blend, the resulting images can be correlated with different local viscoelasticities and also with local variations of stiffness. Although this method is efficient to obtain a material contrast, it is not well adapted to the quantification of mechanical properties.

In the vibrating contact mode achieved with an electrostatic excitation method, ${ }^{9,10}$ the resonance frequency and the quality factor of the cantilever are sensitive to the viscoelastic properties of materials. Both types of information are related to the contact stiffness (itself related to the viscoelastic modulus of the sample surface) and they should be collected to produce quantitative images. This method offers good spatial resolution owing to small penetration depths. In addition, the fact that the cantilever is working at the resonance frequency acts as a supplementary inertial force to increase sensitivity in comparison with (quasi)static methods. However, a difficulty remains with the acquisition time. Recording the spectrum and measuring the resonance frequency and the 


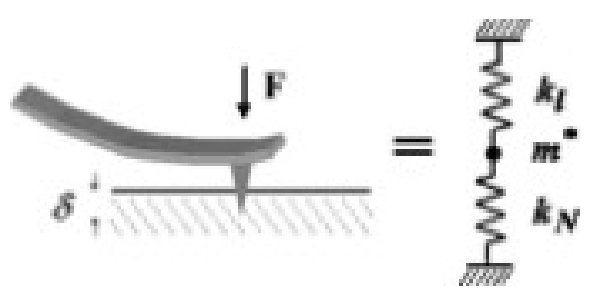

FIG. 1. Spring-mass approximation for the cantilever-tip-sample system. $m_{\text {eff }}$ is the effective mass of both cantilever and tip, $k_{l}$ is the cantilever stiffness, and $k_{N}$ is the sample stiffness.

quality factor is an operation which would require too much time. Several authors have thus proposed resonance tracking systems based on a phase-locked loop. ${ }^{11,12}$

In this article, we propose mathematical image processing of the cantilever vibration parameters (the real and imaginary parts) to directly produce the elastic and damping images, without any significant modification in the atomic force microscope device. As a demonstration, the experimental results on a high-impact polystyrene (HIPS) sample are shown. HIPS is a polymer blend widely used in the automobile industry. It is composed of polystyrene (PS) as the major component and polybutadiene (PB) as the minor component.

\section{THEORETICAL BACKGROUND}

To understand the problem, it is easy to consider that the cantilever-tip-sample system in atomic force microscopy can be approximated by a spring-mass system in the linear elastic regime, as presented in Fig. 1.

When a normal force $F$ is applied on the tip, it penetrates a distance $\delta$ into the sample, which is called the elastic indentation depth. The contact force gradient, also called normal contact stiffness, is given by $k_{n}=\partial F / \partial \delta$. Thus, we can deduce in a first approximation that resonance frequency, given by $f_{0}=(1 / 2 \pi) \sqrt{\left(k_{l}+k_{n}\right) / m_{\text {eff }}}$, is related to the normal contact stiffness.

However, it must be noted that the spring-mass model does not predict exactly the true frequency response of the system. We note, for example, that higher harmonics are not taken into account. A better approximation is obtained with the cantilever beam model coupled with a spring representing the contact stiffness ${ }^{13}$ or a finite element model. ${ }^{9}$

Now, let us analyze the normal contact stiffness. According to the Hertzian contact mechanics theories [Hertz and Derjaquin-Muller-Toporov (DMT)], ${ }^{14,15} k_{n}$ depends not only on the reduced Young's modulus $E^{*}$ but also on the contact radius $a: k_{n}=2 a E^{*}$, with $E^{*}=\left[\left(1-\nu_{t}^{2}\right) / E_{t}+\left(1-\nu_{s}^{2}\right) / E_{s}\right]^{-1} . E_{t}$ and $\nu_{t}$ (respectively, $E_{s}$ and $\nu_{s}$ ) are Young's modulus and Poisson's ratio of the tip (respectively, of the sample). $E^{*}$ is called the reduced modulus. It reflects the simultaneous elastic deformations of the tip and the sample, which are supposed to be isotropic. Knowing $E_{t}$ and $\nu_{t}$, it is possible to deduce the reduced Young's modulus of the sample $E_{s}^{*}$ $=E_{s} /\left(1-\nu_{s}^{2}\right)$. The contact radius verifies $a=\sqrt{R \delta}$, where $R$ is the curvature radius of the tip.

If we consider the response of a linear oscillator to a harmonic excitation, the differential equation describing the motion of the effective mass $m_{\text {eff }}$ of both cantilever and tip is

$$
\begin{aligned}
& \frac{d^{2} \tilde{x}}{d t^{2}}+2 \lambda \frac{d \tilde{x}}{d t}+\omega_{0}^{2} \tilde{x}=\frac{F_{0}}{m_{\mathrm{eff}}} e^{j \omega t}, \\
& \tilde{x}=X_{0} e^{j \omega t} e^{j \varphi},
\end{aligned}
$$

where $\tilde{x}$ is the complex value of the response, $\omega_{0}$ the cantilever-tip-sample system's resonance angular frequency, $\lambda$ the damping coefficient, $F_{0} e^{j \omega t}$ the harmonic excitation force, and $\varphi$ the phase shift between the response and the excitation. The resonance conditions can be found by solving the simplified form of Eq. (1),

$$
X_{0} e^{j \varphi}=\frac{F_{0} / m_{\mathrm{eff}}}{\left(\omega_{0}^{2}-\omega^{2}\right)+2 j \omega \lambda},
$$

where $f_{0}$, the resonance frequency of the system, is given by $f_{0}=\omega_{0} / 2 \pi$, and $f_{1}$, the half-width at half-height of the spectrum, is given by $f_{1}=\lambda / 2 \pi$.

To take into account the transfer function of the electronic detection system, ${ }^{16}$ we introduce an empirical complex constant $C^{*}$,

$$
C^{*}=C e^{j \psi} \text {. }
$$

The detected vibration can be represented by the complex $a+j b=X_{0} e^{j \varphi}$. If we consider that $f$ is close to $f_{0}$, the first order series approximation for expression (3) around $f_{0}$ gives

$$
a+j b=\frac{C^{*}}{f_{0}-f+j f_{1}} .
$$

Relation (5) assumes that the resonance spectrum is a "Lorentzian" curve. If a real part $a$ and an imaginary part $b$ are recorded at a single frequency $f=f_{\text {im }}$ (imaging frequency), from relation (5) we obtain

$$
\begin{aligned}
& f_{0}=f_{\mathrm{im}}+\frac{C(a \cos \psi+b \sin \psi)}{a^{2}+b^{2}}, \\
& f_{1}=\frac{C(a \sin \psi-b \cos \psi)}{a^{2}+b^{2}} .
\end{aligned}
$$

Relations (6) and (7) can be used to produce, respectively, an image of $f_{0}$ and of $f_{1}$. The elastic behavior of the sample can be correlated with $f_{0}$ and its damping behavior with $f_{1}$.

\section{MATERIAL AND METHOD}

In order to test the method, the experimental results on a HIPS sample are shown. HIPS is a polymer blend, composed of PS as the major component and PB as the minor component, that is widely used in the automobile industry. Our sample was a thin film of an $80 \%$ PS-20\% PB blend that was deposited on a silicon wafer substrate. An interesting aspect of this blend is that PS separates from PB, forming spheres at the micrometer scale. The mechanical properties of the two components are known: at ambient temperature PS, being below its $T_{g} \approx 100{ }^{\circ} \mathrm{C}$ (glass transition temperature), has a glassy behavior and $\mathrm{PB}$, being above its $T_{g} \approx-90{ }^{\circ} \mathrm{C}$, has a rubberlike behavior. This would possibly permit us to obtain great modifications in the cantilever's resonance frequency and resonance width, and images were expected with clearly delimited zones. 


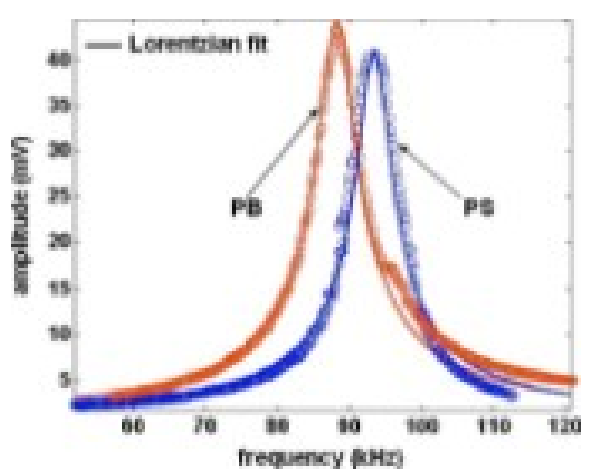

FIG. 2. Typical cantilever's resonance spectra in contact with PS and PB. A Lorentzian fit allows the estimation of the $C^{*}$ constant relative to the equivalent linear oscillator model for the cantilever.

The device we used was a commercial AFM (Autoprobe $\mathrm{CP}$-Park Scientific Instruments) working in contact mode. The commercial V-shaped cantilever (Ultrasharp CSC21A) used for the experiments had a stiffness of $k=0.12 \mathrm{~N} / \mathrm{m}$ and an attached $\mathrm{W}_{2} \mathrm{C}$-coated tip in order to minimize its wear. ${ }^{17}$ The free first resonance frequency was obtained at $19.3 \mathrm{kHz}$.

The vibration was obtained by an electrostatic excitation $^{9,10}$ method and analyzed by a lock-in amplifier. A conductive sample holder, isolated from the piezoelectric tube scanner, was polarized by a sinusoidal potential. The cantilever was attached to the ground and it thus vibrated thanks to an electrostatic pressure actuation. Then, the contact force between the tip and the sample was modulated. The feedback of the microscope maintained the mean contact force $F_{\text {app }}$ constant during the scan. It was thus possible to acquire simultaneously a topographic image. When the excitation frequency was close to the natural frequency of the cantilever, a resonance occurred. It is generally accepted that for small deflections, the photodetector signal is directly proportional to the cantilever deflection. This signal was analyzed by lock-in amplification to extract the real part and the imaginary part of the complex amplitude of vibration. In order to achieve the frequency sweep and resonance spectra acquisitions, an automated measurement was realized using LABVIEW®.

\section{EXPERIMENTAL RESULTS}

As mentioned above, the contact stiffness depends not only on the elastic modulus of the sample but also on the contact radius $a$. When the applied load was increased, $a$ became greater and the resonance frequency increased. We also observed a widening of the curves in such a situation. In the following, all experiments were made at an average applied load of $F_{\text {app }}=0 \mathrm{nN}$. This value was chosen for two reasons: to obtain comparable measurements (and no applied load-dependent measurements) and to optimize spatial resolution.

First of all, we determined the empirical complex constant $C^{*}=C e^{j \psi}$. The cantilever's resonance spectra in contact with PS and PB were recorded, as shown in Fig. 2. We measured $f_{0_{\mathrm{PS}}}=93.4 \mathrm{kHz}, f_{1_{\mathrm{PS}}}=1.7 \mathrm{kHz}, f_{0_{\mathrm{PB}}}=88.2 \mathrm{kHz}$, and $f_{1_{\mathrm{PB}}}=2.5 \mathrm{kHz}$. The experimental amplitude $X_{0}$ vs frequency $f$ was fitted by a Lorentzian curve according to relation (5),

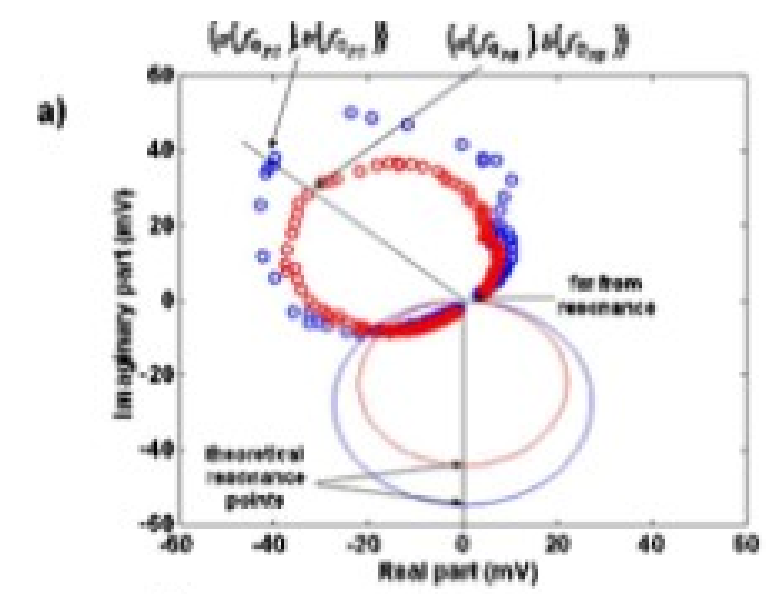

b)

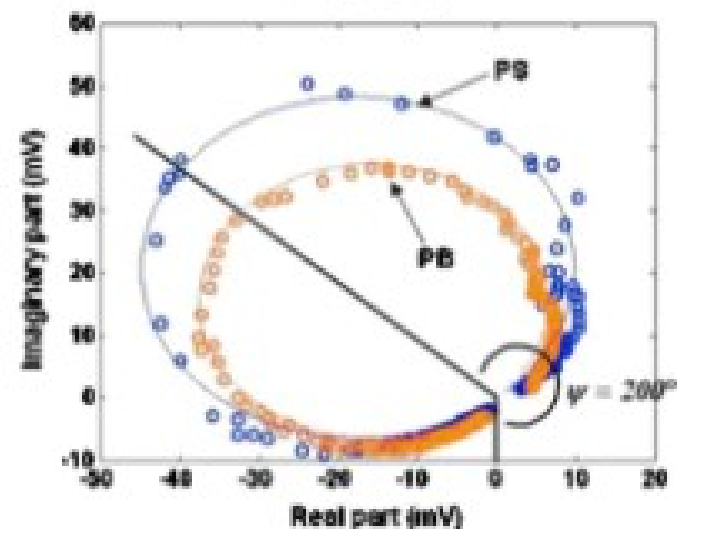

FIG. 3. (a) Experimental and theoretical points $[a(f), b(f)]$ describing a circle. Far from resonance, the points tend toward the never reached point $(0,0)$. Near resonance, $[a(f), b(f)]$ is always diametrically opposed to $(0,0)$. (b) Experimental points are correctly fitted by the theoretical circle after a rotation from an angle $\psi=200^{\circ}$. Such a correction is necessary to take into account the electronics' transfer function of the system.

$$
X_{0}(f)=\left|\frac{C}{f_{0}-f+j f_{1}}\right|
$$

Adjustments for the two materials agreed for $C \approx 9.5 \times 10^{4}$. In the complex plane, the points $[a(f), b(f)]$ are given by relation (5). They describe a circle. Far from resonance, these points theoretically tended towards the never-reached origin point $(0,0)$, i.e., the circle was never closed. At resonance, $\left[a\left(f_{0}\right), b\left(f_{0}\right)\right]$ was always diametrically opposed to the origin. Without taking into account the electronics' transfer function of the system, we had $a\left(f_{0}\right)+j b\left(f_{0}\right)=-j C / f_{1}$. Thus, $\left[a\left(f_{0}\right), b\left(f_{0}\right)\right]$ was located on the ordinate axis. Practically, when the electronics' transfer function was taken into account, $a\left(f_{0}\right)+j b\left(f_{0}\right)$ was no longer imaginary and we generally observed a rotation of the theoretical circle by an angle $\psi$. The theoretical and experimental complex plane circles are presented for comparison in Fig. 3(a). For our application, experimental points were fitted with $\psi=200^{\circ}$, as shown in Fig. 3(b). Then, we obtained $C^{*} \approx 9.5 \times 10^{4} e^{j 200}$. Figures 4(a) and 4(b) are, respectively, images of the real part $a$ and the imaginary part $b$ at an intermediate imaging frequency $f_{\text {im }}=92 \mathrm{kHz}$ between $f_{o_{\mathrm{PB}}}$ and $f_{0_{\mathrm{PS}}}$. The investigated zone $\left(20 \times 20 \mu \mathrm{m}^{2}\right)$ is representative of the material structure at the micrometer scale. 

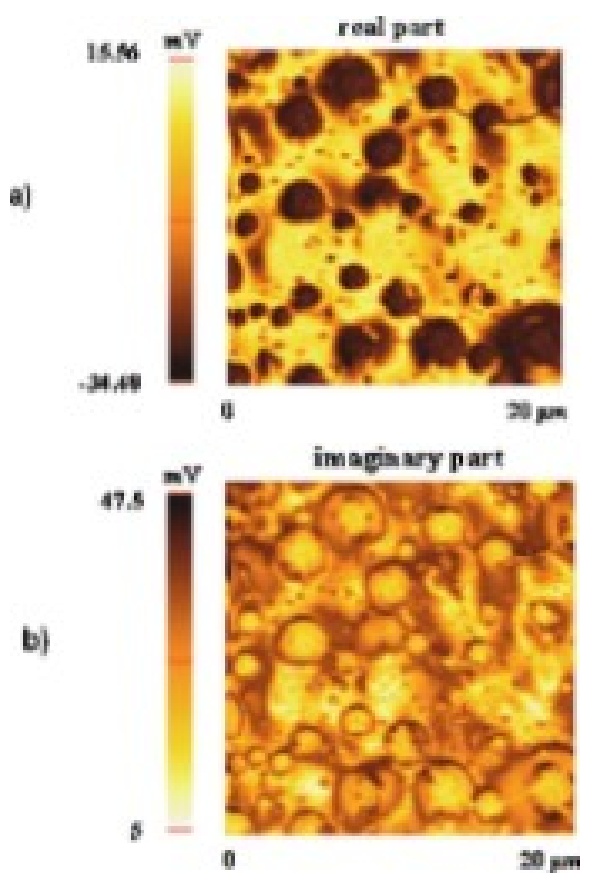

FIG. 4. Images of real part $a$ and imaginary part $b$ of the cantilever's vibration at frequency $f_{\mathrm{im}}=92 \mathrm{kHz}$.

According to relations (6) and (7), processing of both the real and imaginary part images was performed. Images of resonance frequency $f_{0}$ and half-width at half-height $f_{1}$ were produced. The contrast of the images was related to the sample frequency-dependent viscoelastic behavior at the imaging frequency $f_{\mathrm{im}}$. Indeed, resonance frequency depends on contact stiffness, whereas half-width at half-height depends, partially, on the material damping.

In Fig. 5(a), the dark zones correspond to the more com-

a)
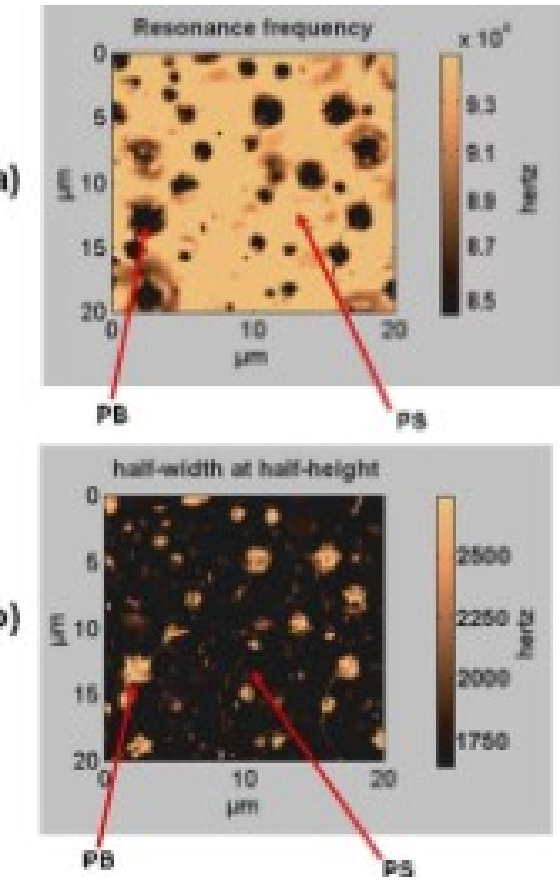

FIG. 5. Resonance frequency and half-width at half-height images obtained by processing of the real and imaginary part images.

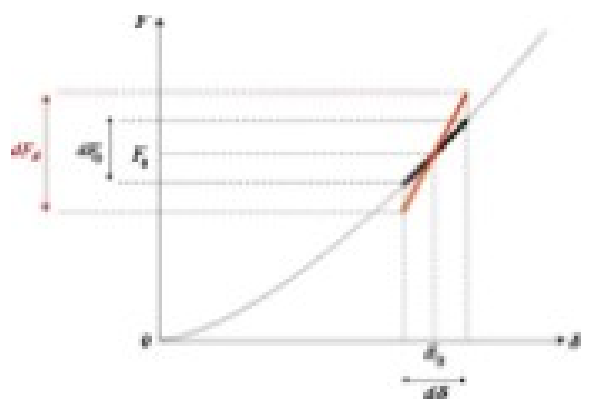

FIG. 6. The contact modulation seen as a small variation of indentation $d \delta$ around a mean value $\delta_{0}$. The contact force, represented according to Hertz theory, is the sum of static component $F_{s}$ and a dynamical component $d F_{d}$.

pliant material while the lighter zones correspond to the stiffer. In Fig. 5(b), the dark zones correspond to the less dissipative material while the lighter zones correspond to the most dissipative.

\section{ON THE QUANTIFICATION OF THE VISCOELASTIC MODULUS}

We will now discuss the quantification of mechanical properties. The produced images were used to estimate a reduced complex viscoelastic modulus, expressed as $E^{\prime *}\left(f_{\text {im }}\right)+j E^{\prime \prime *}\left(f_{\text {im }}\right)$. The in-phase component $E^{\prime *}\left(f_{\text {im }}\right)$ was called the storage modulus. The out-of-phase component $E^{\prime \prime}\left(f_{\text {im }}\right)$ was called the loss modulus. The quantification of these moduli was made possible by applying contact mechanics theories. ${ }^{14,15,18-20}$ We underline the fact that, because technical data on beam and tip sizes are not reliable enough, uncertainty will always be substantial.

Let us consider the case of a vibrating tip-sample contact. It what follows, we were inspired by the method proposed by Mahaffy et $a l^{21}$ The relation between the tip penetration and the applied load in a static regime was given by

$$
F=\frac{4}{3} E^{*} R^{1 / 2} \delta^{3 / 2}
$$

We then considered a small variation of the tip penetration $d \delta$ around a mean (static) value $\delta_{0}$, as shown in Fig. 6 . The tip is supposed to be infinitely stiff with respect to the sample. The first order series approximation for expression (9) around $\delta_{0}$ gave

$$
F \approx \frac{4}{3} R^{1 / 2}\left(E_{0}^{*} \delta_{0}^{3 / 2}+\frac{3}{2} E_{d}^{*} \delta_{0}^{1 / 2} d \delta\right)=F_{0}+d F_{d},
$$

where $E_{0}^{*}$ is the static-reduced elastic modulus of the sample. $E_{d}^{*}=E^{\prime *}+j E^{\prime \prime *}$ is the dynamical viscoelastic modulus of the sample at the imaging frequency $f_{\mathrm{im}} \cdot d F_{d}$ is the force variation induced by $d \delta$. In Fig. 6 , this variation is presented in comparison with the variation $d F_{0}$ which would have been induced if the dynamical properties of the sample had been the same as the static ones. In the case of polymers, the static behavior interferes with the dynamical behavior. For most polymers, $E^{\prime *}>E_{0}^{*}$, thus we have $d F_{d}>d F_{0}$.

The selection of a viscous damping model in Eq. (1) was primarily used for ease of analysis. However, the behavior of a viscoelastic material is better described through the use of 
TABLE I. Experimental values for resonance frequency and half-width of resonance curve, calculated complex contact stiffness, and viscoelastic modulus for PS and PB.

\begin{tabular}{lccllll}
\hline \hline & $\begin{array}{c}f_{0} \\
(\mathrm{kHz})\end{array}$ & $\begin{array}{c}f_{1} \\
(\mathrm{kHz})\end{array}$ & $\begin{array}{l}k_{N_{\mathrm{DMT}}}^{\prime} \\
(\mathrm{N} / \mathrm{m})\end{array}$ & $\begin{array}{l}k_{N_{\mathrm{DMT}}}^{\prime \prime} \\
(\mathrm{N} / \mathrm{m})\end{array}$ & $\begin{array}{c}E^{{ }^{*}} \\
(\mathrm{GPa})\end{array}$ & $\begin{array}{c}E^{\prime \prime} \\
(\mathrm{GPa})\end{array}$ \\
\hline $\mathrm{PS}$ & $94 \pm 3$ & $1.75 \pm 0.2$ & $2.74^{\mathrm{a}}$ & $0.1^{\mathrm{a}}$ & $0.29^{\mathrm{a}}$ & $0.025^{\mathrm{a}}$ \\
$\mathrm{PB}$ & $86 \pm 3$ & $2.75 \pm 0.2$ & $2.22^{\mathrm{a}}$ & $0.15^{\mathrm{a}}$ & $0.01^{\mathrm{a}}$ & $0.0016^{\mathrm{a}}$ \\
\hline \hline
\end{tabular}

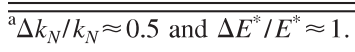

the hysteretic model ${ }^{22}$ in which damping is represented by a complex stiffness $k_{N}^{\prime}+j k_{N}^{\prime \prime}$. We can deduce from expression (10) that the normal contact stiffness in the case of a Hertzian contact without adhesion can be expressed as

$$
k_{N_{\mathrm{Hertz}}}^{\prime}+j k_{N_{\mathrm{Hertz}}}^{\prime \prime}=\frac{d F_{d}}{d \delta}=2 R^{1 / 2} \delta_{0}^{1 / 2} E_{d}^{*} .
$$

Introducing the first term of Eq. (10) into Eq. (12), we obtained

$$
k_{N_{\text {Hertz }}}^{\prime}=E^{\prime *}\left(\frac{6 R F_{0}}{E_{0}^{*}}\right)^{1 / 3},
$$

and

$$
k_{N_{\text {Hertz }}^{\prime \prime}}^{\prime \prime}=E^{\prime \prime} *\left(\frac{6 R F_{0}}{E_{0}^{*}}\right)^{1 / 3} .
$$

To take into account the contact in presence of adhesion, we used the DMT ${ }^{15}$ model. This model is just an offset of the Hertz model by the adhesive force $F_{\text {ad. }}$. Then, we deduced

$$
k_{N_{\mathrm{DMT}}}^{\prime}=E^{\prime *}\left[\frac{6 R\left(F_{0}+F_{\mathrm{ad}}\right)}{E_{0}^{*}}\right]^{1 / 3},
$$

and

$$
k_{N_{\mathrm{DMT}}}^{\prime \prime}=E^{\prime \prime} *\left[\frac{6 R\left(F_{0}+F_{\mathrm{ad}}\right)}{E_{0}^{*}}\right]^{1 / 3} .
$$

The resonance conditions verified the modified form of relation (5), where $f_{0}$ and $f_{1}$, respectively, the contact resonance frequency and half-width at half-height, were given by

$$
f_{0}=\frac{1}{2 \pi} \sqrt{\frac{k_{N_{\mathrm{DMT}}^{\prime}}+k_{l}}{m_{\mathrm{eff}}}},
$$

and

$$
f_{1}=\frac{k_{N_{\text {DMT }}^{\prime \prime}}}{8 \pi^{2} m_{\text {eff }} f_{0}} .
$$

We next tried to quantify the viscoelastic properties of the HIPS sample. Experiments were conducted with the following parameters, which were inserted into our numerical calculations: $R \approx 50 \mathrm{~nm}, F_{0} \approx 0 \mathrm{nN}$, and $F_{\text {ad }} \approx 10 \mathrm{nN}$. $F_{\text {ad }}$ was obtained by measuring the pull-off force and was approximately the same on PS and PB. The static moduli of PS and $\mathrm{PB}$ were those measured by Tsukruk and Huang: ${ }^{23} E_{0}(\mathrm{PS})$ $=3 \mathrm{GPa}, \quad v_{0}(\mathrm{PS})=0.33$ and $E_{0}(\mathrm{~PB}) \approx 0.003 \mathrm{GPa}, \quad v_{0}(\mathrm{~PB})$ $\approx 0.5$. The corresponding reduced moduli were $E_{0}^{*}(\mathrm{PS})$ $\approx 3.4 \mathrm{GPa}$ and $E_{0}^{*}(\mathrm{~PB})=4 \mathrm{MPa}$. We measured the average values for $f_{0}$ and $f_{1}$ (Table $\mathrm{I}$ ). Knowing the free resonance frequency $f=19.3 \mathrm{kHz}, k_{l}=0.12 \mathrm{~N} / \mathrm{m}$ and considering that the contact stiffness is equal to zero, it was possible to deduce the effective mass $m_{\text {eff }}$ using relation (16): $m_{\text {eff }} \approx 8.2$ $\times 10^{-12} \mathrm{~kg}$. Using Eqs. (16) and (17), we estimated $k_{N_{\text {DMT }}^{\prime}}$ and $k_{N_{\mathrm{DMT}}}^{\prime \prime}$ for PS and PB at the imaging frequency (Table I). Finally, using (14) and (15) we obtained the values of $E^{\prime *}$ and $E^{\prime \prime *}$ in the vicinity of the imaging frequency $(92 \mathrm{kHz})$ (Table I). The result for PS seemed to be underestimated, while the value for $\mathrm{PB}$ was more coherent. Indeed, at the imaging frequency PB probably reached the glassy behavior. PS was expected to remain on the glassy plateau with a storage modulus comparable to the static one. The calculated modulus was, however, approximately ten times smaller than the expected value. If we try to explain this underestimation by evaluating all uncertainties $(\Delta R / R \approx 0.5, \Delta F / F \approx 0.1$, $\Delta k_{l} / k_{l} \approx 0.5$, etc.), we find systematic errors $\Delta k_{N} / k_{N} \approx 0.5$ and $\Delta E^{*} / E^{*} \approx 1$. These very important errors are not sufficient to explain the difference between the actual value and the expected one. A possible explanation is that the cantilever used for this experiment was not well adapted for testing stiffness in the range of PS or above. As mentioned by Rabe et al., ${ }^{24}$ cantilever stiffness must verify $k_{N} / k_{l} \leqslant 10$ to be sufficiently sensitive to mechanical properties. Moreover, the spring-mass model predicts the true frequency response of the cantilever only in this condition. This condition was not respected in the present study of PS and the spring-mass model was no longer adequate. For this reason, we remain careful about announcing a quantitative elastic modulus. ${ }^{25}$

\section{DISCUSSION}

In this article we presented a new method to separate the elastic and damping information of a viscoelastic sample by resonance frequency mapping in atomic force modulation microscopy. This method is based on the measurement of the real and imaginary parts of the cantilever's vibration at a single frequency. Numerical image processing allowed us to produce resonance frequency and resonance curve width images on a high-impact polystyrene sample. We demonstrated that these experimental data are theoretically related to the sample's storage modulus and loss modulus, respectively. The quantification of images in terms of viscoelastic moduli is thus possible but is still dependent on the reliability of the sensor's technical characteristics.

${ }^{1}$ P. Maivald, H. J. Butt, S. A. C. Gould, C. B. Prater, B. Drake, J. A. Gurley, V. B. Elings, and P. K. Hansma, Nanotechnology 2, 103 (1991).

${ }^{2}$ S. N. Magonov, V. Elings, and M.-H. Whangbo, Surf. Sci. Lett. 375, 385 (1997).

${ }^{3}$ Force Volume Imaging, Application Note, Digital Instruments, Veeco Metrology Group (www.veeco. com).

${ }^{4}$ C. Reynaud, F. Sommer, C. Quet, N. El Bounia, and T. Minh Duc, Surf. Interface Anal. 30, 185 (2000).

${ }^{5}$ M. Heuberger, G. Dietler, and L. Schlapbach, Nanotechnology 5, 12 (1994).

${ }^{6}$ S. A. Syed Asif, K. J. Wahl, R. J Colton, and O. L. Warren, J. Appl. Phys. 90, 1192 (2001).

${ }^{7}$ NanoDMA ${ }^{\mathrm{TM}}$ viscoelastic materials, Hysitron (www.hysitron. com).

${ }^{8}$ B. Anczykowski, B. Gotsmann, H. Fuchus, J. P. Cleveland, and V. B. Elings, Appl. Surf. Sci. 140, 376 (1999).

${ }^{9}$ R. Arinéro and G. Lévêque, Rev. Sci. Instrum. 74, 104 (2003).

${ }^{10}$ S. Cuénot, C. Frétigny, S. Demoustier-Champagne, and B. Nysten, J. Appl. Phys. 93, 5650 (2003).

${ }^{11}$ K. Yamanaka, Y. Maruyama, T. Tsuji, and K. Nakamoto, Appl. Phys. Lett. 
78, 1939 (2001).

${ }^{12}$ K. Kobayachi, H. Yamada, and K. Matsushige, Surf. Interface Anal. 33, 89 (2002).

${ }^{13}$ K. Yamanaka and S. Nakano, Jpn. J. Appl. Phys., Part 1 35, 3787 (1996).

${ }^{14}$ H. Hertz, J. Reine Angew. Math. 92, 156 (1882).

${ }^{15}$ B. V. Derjaguin, V. M. Muller, and Y. P. Toporov, J. Colloid Interface Sci. 53, 314 (1975).

${ }^{16}$ M. Stark, R. Guckenberger, A. Stemmer, and R. W. Stark, J. Appl. Phys. 98, 114904 (2005)

${ }^{17}$ AFM probes, Micromasch (www.spmtips.com).

${ }^{18}$ K. L. Johnson, Contact Mechanics (Cambridge University Press, Cambridge, 1985).
${ }^{19}$ D. Maugis, Contact, Adhesion and Rupture of Elastic Solids (Springer, Berlin, 2000).

${ }^{20}$ O. Pietrement and M. Troyon, J. Colloid Interface Sci. 226, 166 (2000).

${ }^{21}$ R. E. Mahaffy, C. K. Shih, F. C. MacKintosh, and J. Käs, Phys. Rev. Lett. 85, 880 (2000)

${ }^{22}$ Y. M. Haddad, Viscoelasticity of Engineering Materials, (Chapman and Hall, London, 1995).

${ }^{23}$ V. V. Tsukruk and Z. Huang, Polymer 41, 5541 (2000).

${ }^{24}$ U. Rabe, K. Janser, and W. Arnold, Rev. Sci. Instrum. 67, 3281 (1996).

${ }^{25} \mathrm{~B}$. Clair, R. Arinéro, G. Lévêque, M. Ramonda, and B. Thibaut, IAWA Journal (International Journal of Wood Anatomists), 24, 223 (2003). 